\title{
O processo de glaciamento associado a antioxidantes naturais melhora a conservação
}

\section{de filés de tilápia do Nilo}

The glazing process associated with natural antioxidants improves the conservation of Nile tilapia

fillets

El proceso de glaciación asociado a los antioxidantes naturales mejora la conservación de los filetes de tilapia del Nilo

\section{Resumo}

O objetivo deste estudo foi avaliar compostos antioxidantes no processo de glaciamento para a conservação de filés de tilápia. Para isso, foram testados quatro tratamentos: controle (sem adição de antioxidantes), butil-hidroxi-tolueno (BHT), cúrcuma e $\beta$-caroteno. A adição dos antioxidantes foi feita no momento do glaciamento. Em seguida, os filés foram refrigerados durante 10 dias. Foram realizadas análises de composição centesimal, bioquímicas e de peroxidação lipídica no quinto dia e ao final dos experimentos (décimo dia). $\mathrm{O}$ uso de cúrcuma ou de $\beta$-caroteno resultou numa menor quantidade de amônia $(1,06 \pm 0,10$ e 1,42 $\pm 0,07 \mu \mathrm{mol} / \mathrm{g}$, respectivamente), indicando um processo de putrefação menos acelerado, comparativamente ao uso de BHT $(2,32 \pm 0,09)$. Na análise de substâncias reativas ao ácido tiobarbitúrico houve um decréscimo, em torno de 50\%, nos filés submetidos aos tratamentos com cúrcuma ou $\beta$ caroteno $(1,71 \pm 0,51$ e $1,87 \pm 0,32 \mathrm{nmol} \mathrm{MDA} / \mathrm{mg}$ proteína, respectivamente) comparados ao controle (sem antioxidante). Os resultados comprovam a importância do uso de antioxidantes, como cúrcuma e $\beta$-caroteno, que agiram melhorando a conservação (diminuindo a ação de espécies reativas e a putrefação), contribuindo para uma melhor qualidade físico-química do filé. Recomenda-se a utilização de antioxidantes no glaciamento dos filés, como o $\beta$-caroteno e a cúrcuma, que, por serem naturais, seriam melhores opções para a utilização no processamento do pescado quando comparados à produtos sintéticos.

Palavras-chave: $\beta$-caroteno; Cúrcuma; Oreochromis niloticus; Processamento de peixes. 


\begin{abstract}
The aim of this study was to evaluate antioxidant compounds in the glazing process for the conservation of tilapia fillets. For this, four treatments were tested: control (without addition of antioxidants), butyl-hydroxy-toluene (BHT), turmeric and $\beta$-carotene. The addition of antioxidants was done along with the glazing process, then the fillets were refrigerated for 10 days. Proximate composition, biochemical and lipid peroxidation analyzes were performed on the fifth day and at the end of the experiments (tenth day). The use of turmeric or $\beta$-carotene resulted in a lower amount of ammonia $(1.06 \pm 0.10$ and $1.42 \pm 0.07 \mu \mathrm{mol} / \mathrm{g}$, respectively), indicating a lower putrefaction process, compared to the use of BHT (2.32 \pm 0.09$)$. In the analysis of TBARS there was a decrease, around 50\%, in fillets submitted to treatments with turmeric or $\beta$-carotene $(1.71 \pm 0.51$ and $1.87 \pm 0.32 \mathrm{nmol} \mathrm{MDA} / \mathrm{mg}$ protein, respectively) compared to the control (without antioxidant). The results prove the importance of using antioxidants, such as turmeric and $\beta$ carotene, which acted by improving conservation (decreasing the action of reactive species and putrefaction), contributing to a better physicochemical quality of the fillet. The use of antioxidants in the glazing of fillets is recommended, such as $\beta$-carotene and turmeric, which, as they are natural, could be better options for use in fish processing when compared to synthetic products.
\end{abstract}

Keywords: $\beta$-carotene; Turmeric; Oreochromis niloticus; Fish processing.

\title{
Resumen
}

El objetivo de este estudio fue evaluar compuestos antioxidantes en el glaseado para la conservación de filetes de tilapia. Para ello, se probaron cuatro tratamientos: control (sin adición de antioxidantes), butil-hidroxi-tolueno (BHT), cúrcuma y $\beta$-caroteno. La adición de antioxidantes se realizó en el momento del proceso del glaseado, luego los archivos se refrigeraron durante 10 días. Los análisis de composición próxima, bioquímica y de peroxidación lipídica se realizaron al quinto día y al final del experimento (décimo día). El uso de cúrcuma o $\beta$-caroteno resultó en una menor cantidad de amoníaco $(1,06 \pm 0,10$ y $1,42 \pm 0,07 \mu \mathrm{mol} / \mathrm{g}$, respectivamente), lo que indica un proceso de putrefacción menos acelerado, en comparación con el uso de BHT (2,32 \pm 0.09$)$. En el análisis de TBARS hubo una disminución, alrededor del $50 \%$, en los filetes sometidos a tratamientos con cúrcuma o $\beta$-caroteno $(1,71 \pm 0,51$ y 1,87 $\pm 0,32 \mathrm{nmol} \mathrm{MDA} / \mathrm{mg}$ proteína, respectivamente) en comparación con control (sin antioxidante). Los resultados demuestran la importancia del uso de antioxidantes, como la cúrcuma y el $\beta$-caroteno, que actuaron mejorando la conservación (disminuyendo la acción de las especies reactivas y la putrefacción), contribuyendo a una mejor calidad físico química del filete. Se recomienda el uso de antioxidantes en el glaseado de filetes, como el $\beta$-caroteno y la cúrcuma, que por ser naturales serían mejores opciones para su uso en el procesamiento del pescado en comparación con los productos sintéticos.

Palabras clave: $\beta$-caroteno; Cúrcuma; Oreochromis niloticus; Procesamiento de pescado.

\section{Introdução}

O consumo de peixe está associado a benefícios à saúde, devido ao conteúdo proteico com elevado valor biológico e vários outros nutrientes importantes, como ácidos graxos (Beraldo et al., 2020; Gonçalves et al., 2020; Nascimento \& Scalabrini, 2020; Soares et al., 2016). O alto valor nutricional do peixe contribuiu para que nos últimos anos a demanda por este produto aumentasse, especialmente para quem deseja adotar uma alimentação mais saudável (FAO, 2020; Nascimento \& Scalabrini, 2020; Silva et al., 2020; Soares et al., 2016; Wu et al., 2019). O consumo de peixes e demais pescados e seus derivados no Brasil ainda é baixo (10 kg/hab/ano) comparado à média mundial (20 kg/hab/ano). No entanto, o país é o quarto maior produtor de tilápias do mundo, o principal peixe produzido hoje no país (Schreiber et al., 2021).

Embora a carne de peixe seja rica em nutrientes, é extremamente perecível, devido à sua composição físico-química. Fatores como elevado teor proteico, presença de enzimas proteolíticas, alta atividade de água e pH próximo à neutralidade intensificam o processo de degradação (Prabhakar et al., 2020; Wu et al., 2019). Por isto, torna-se indispensável a adoção de estratégias para evitar a perda da qualidade nutritiva do peixe.

No processo de beneficiamento de peixes, as indústrias utilizam o processo de glaciamento. Este processo consiste na aplicação de água, por imersão ou pulverização, seguido de congelamento para que seja formada uma camada de gelo que evita o contato do peixe com o ar atmosférico. Isto retarda a perda de umidade e a oxidação lipídica (Neiva et al., 2018).

Outro procedimento industrial utilizado para retardar reações químicas indesejadas é o uso de antioxidantes (Silva et al., 2019; Viana \& Arenari, 2019), estes podem estar associados a outros processos industriais de beneficiamento de peixes, como defumação (Matiucci et al., 2021). 
A adição de antioxidantes no processo de glaciamento representa uma alternativa para aumentar a vida útil dos filés. Os antioxidantes são compostos capazes de neutralizar as espécies reativas, inibindo ou diminuindo a formação de outros compostos prejudiciais à qualidade do produto (Silva et al., 2019; Viana \& Arenari, 2019). Antioxidantes sintéticos como o BHA (butylated hydroxyanisole), o BHT (butylhydroxytoluene) e o TBHQ (tert-butylhydroquinone) têm sido comumente usados. O BHT é efetivo, no entanto, possui um odor desagradável em temperatura elevada e alguns apresentam uso restrito em muitos países devido à possibilidade de causar efeitos indesejáveis (Silva et al., 2019). Esforços têm sido feitos para utilizar antioxidantes naturais, mantendo ainda mais a característica de alimentos saudáveis (Alves et al., 2020; Dias et al., 2018; Machado et al., 2020). Dentre os antioxidantes naturais empregados estão a cúrcuma (Curcuma longa), com propriedades antioxidantes e anti-inflamatórias (Marchi et al., 2016). Outro aditivo natural utilizado é a vitamina A, que nos alimentos de origem vegetal é encontrada como pró-vitamina A ou carotenoides, cujo principal exemplo é o $\beta$-caroteno (Mesquita \& Torquilho, 2016).

Neste estudo, objetivou-se avaliar o potencial antioxidante de dois compostos naturais (cúrcuma e $\beta$-caroteno) e um sintético (BHT) adicionados no processo de glaciamento para a conservação de filés de tilápia.

\section{Metodologia}

\section{Amostras e ensaio experimental}

Os filés frescos de tilápia (Oreochromis niloticus) foram adquiridos de um frigorífico de pequeno porte certificado pelo Serviço de Inspeção Municipal, localizado na região noroeste do Rio Grande do Sul, Brasil. O antioxidante BHT (PA) foi adquirido virtualmente (Synth, Brasil), a cúrcuma (em pó), em loja de condimentos e especiarias, no município Palmeira das Missões, RS, e o $\beta$-caroteno (grau farmacêutico), em laboratório de manipulação no município Frederico Westphalen, RS. A quantidade utilizada em cada tratamento está de acordo com o estabelecido pela Agência Nacional de Vigilância Sanitária (Anvisa), sendo: 0,002\% para antioxidantes naturais e 0,01\% para o sintético para cada $100 \mathrm{~g}$ de amostra (Anvisa, 1998).

Os filés foram pesados e divididos em porções de $100 \mathrm{~g}$ para cada tratamento. Em seguida, foram submetidos aos tratamentos: controle (sem adição de antioxidantes), BHT, cúrcuma e $\beta$-caroteno. Os antioxidantes foram adicionados no momento de imersão dos filés, no processo de glaciamento (Neiva et al., 2018). Após o processamento, foram mantidos refrigerados à temperatura de -2 a $2{ }^{\circ} \mathrm{C}$ para posterior análise, aos 5 e 10 dias, que corresponde ao tempo de prateleira do filé refrigerado para o consumo (Vasconcelos \& Melo, 2016).

\section{Análises bromatológicas}

A análise da composição físico-química dos filés foi feita de acordo com as metodologias recomendadas pela Association of Official Agricultural Chemists (AOAC, 2005) para umidade, matéria mineral e proteína bruta (N x 6,25), de acordo com os métodos $925.09,923.03$ e 920.87 , respectivamente. A gordura foi extraída e quantificada pelo procedimento estabelecido por Bligh e Dyer (1959).

\section{Análises bioquímicas}

Os tecidos foram homogeneizados conforme descrito por Azambuja et al. (2011). As determinações foram feitas por espectrofotometria. A peroxidação lipídica foi estimada pelas substâncias reativas ao ácido tiobarbitúrico [thiobarbituric acid reactive substances (TBARS)] (Buege \& Aust, 1978), com geração de um complexo róseo ( $\lambda=535 \mathrm{~nm}$ ), formado pela reação de malondialdeído (MDA) com o ácido. A quantificação de glicose foi feita utilizando kit comercial enzimático, que emprega glicose oxidase e peroxidase e forma um produto de coloração vermelha $(\lambda=505 \mathrm{~nm})$. O glicogênio foi determinado usando o 
método de Dubois et al. (1956), utilizando fenol e ácido sulfúrico, que reage com a glicose (derivada da hidrólise do polímero), gerando um composto alaranjado $(\lambda=480 \mathrm{~nm})$. A amônia total foi quantificada pela técnica do salicilato (Verdouw et al., 1978), produzindo uma coloração verde $(\lambda=595 \mathrm{~nm})$, cuja intensidade de cor é proporcional ao teor de amônia na amostra.

\section{Análises estatísticas}

Os dados, em triplicata, foram submetidos à análise de ANOVA e, ao apresentarem significância $(p<0,05)$, as médias foram comparadas pelo teste de Duncan. Os procedimentos analíticos foram realizados com a utilização do pacote estatístico Statistical Analysis System Studio ${ }^{\circledR}$ (https://odamid-usw2.oda.sas.com/SASStudio).

\section{Resultados e Discussão}

A análise da composição físico-química dos filés submetidos aos diferentes tratamentos é apresentada na Tabela 1. Os filés tratados com $\beta$-caroteno apresentaram maior quantidade de proteína em relação aos demais tratamentos e similar aquele do tratamento com BHT. O mesmo tratamento apresentou menor umidade, corroborando com Oliveira et al. (2008) que observaram que a perda de água determinou uma maior concentração de proteína nos filés de tilápia do Nilo. A utilização de cúrcuma proporcionou umidade ao filé, similar àquelas apresentadas pelos grupos controle e BHT. A maior umidade significa uma maior retenção de água, melhorando a preservação das células musculares, conferindo à carne uma maior suculência (Góes-Favon et al., 2021; Oliveira et al., 2019; Souza et al., 2020).

Tabela 1. Parâmetros físico-químicos de filés de tilápia submetidos ao glaciamento com o uso de antioxidantes.

\begin{tabular}{|c|c|c|c|c|c|}
\hline \multirow[b]{2}{*}{$\begin{array}{c}\text { Parâmetro } \\
(\%)\end{array}$} & \multicolumn{5}{|c|}{ Tratamento } \\
\hline & Controle & Cúrcuma & $\beta$-caroteno & BHT & $p$-valor \\
\hline \multicolumn{6}{|c|}{5 dias de resfriamento } \\
\hline Proteína bruta & $15,49 \pm 0,99^{b}$ & $15,32 \pm 0,76^{\mathrm{b}}$ & $17,32 \pm 1,94^{\mathrm{a}}$ & $18,02 \pm 0,73^{\mathrm{a}}$ & 0,0017 \\
\hline Umidade & $79,90 \pm 1,27^{\mathrm{a}}$ & $79,44 \pm 0,205^{\mathrm{ab}}$ & $76,60 \pm 0,094^{c}$ & $77,93 \pm 1,40^{\mathrm{bc}}$ & 0,0036 \\
\hline Gordura & $5,91 \pm 0,62$ & $4,95 \pm 1,34$ & $5,05 \pm 1,67$ & $4,51 \pm 1,64$ & NS \\
\hline Matéria mineral & $1,43 \pm 0,30$ & $1,32 \pm 0,38$ & $1,29 \pm 0,03$ & $1,33 \pm 0,18$ & NS \\
\hline \multicolumn{6}{|c|}{10 dias de resfriamento } \\
\hline Proteína bruta & $15,94 \pm 1,38$ & $16,17 \pm 1,38$ & $16,35 \pm 2,44$ & $18,28 \pm 1,39$ & NS \\
\hline Umidade & $81,35 \pm 1,00$ & $79,74 \pm 1,33$ & $79,25 \pm 3,43$ & $78,65 \pm 1,12$ & NS \\
\hline Gordura & $3,80 \pm 0,62$ & $4,55 \pm 1,17$ & $4,21 \pm 1,64$ & $4,17 \pm 1,46$ & NS \\
\hline Matéria mineral & $1,17 \pm 0,12^{\mathrm{a}}$ & $0,98 \pm 0,14^{\mathrm{ab}}$ & $0,76 \pm 0,12^{\mathrm{b}}$ & $1,18 \pm 0,22^{\mathrm{a}}$ & 0,0123 \\
\hline
\end{tabular}

Valores expressos como média \pm desvio padrão. Tratamentos com diferentes letras na mesma linha diferem significativamente de acordo com o teste de Duncan ( $p<0,05)$. NS: não significativo. Fonte: Autores (2021).

Em relação à composição físico-química ao décimo dia de armazenamento, houve diferença significativa apenas para a matéria mineral. Os filés com adição de $\beta$-caroteno apresentaram menor quantidade de matéria mineral quando comparados ao quinto dia de armazenamento. A explicação para a menor quantidade de mineral pode ser devido ao descongelamento, quando grande quantidade de fluidos são eliminados, provocando a perda de nutrientes, dentre eles, sais minerais (Vasconcelos \& Melo, 2016). Os valores de matéria mineral apresentados no trabalho são comparáveis aos encontrados por Olopade et al. (2016) e Colpini et al. (2017) em filés de tilápia, inferiores a 1,5\%. Os demais valores de composição centesimal observados no presente estudo são semelhantes aos determinados por outros autores para a mesma espécie (Alves et al., 2020; Moura et al., 2018).

Os parâmetros bioquímicos analisados apresentaram diferença significativa em ambos os períodos de refrigeração (Tabela 2). Os valores de TBARS foram maiores aos cinco dias, ou seja, decrescem com o decorrer do tempo. Isto sugere que 
o pico da degradação lipídica, cujo subproduto é o MDA, ocorre nos primeiros dias de armazenamento. Os tratamentos com antioxidantes conferiram às amostras níveis de TBARS inferiores aos do controle, independentemente do prazo de estocagem. Isto comprova esta habilidade química das substâncias testadas para tratar o alimento aqui empregado, filés de peixe, que agem neutralizando as consequências das espécies reativas, diminuindo a peroxidação lipídica (Battisti et al., 2017; Machado et al., 2020).

Tabela 2. Parâmetros bioquímicos de filés de tilápia submetidos ao glaciamento com o uso de antioxidantes.

\begin{tabular}{|c|c|c|c|c|c|}
\hline \multirow[b]{2}{*}{ Parâmetro } & \multicolumn{5}{|c|}{ Tratamento } \\
\hline & Controle & Cúrcuma & $\beta$-caroteno & BHT & $p$-valor \\
\hline \multicolumn{6}{|c|}{5 dias de resfriamento } \\
\hline Amônia $(\mu \mathrm{mol} / \mathrm{g})$ & $1,86 \pm 0,23^{\mathrm{c}}$ & $1,55 \pm 0,10^{\mathrm{c}}$ & $2,68 \pm 0,16^{\mathrm{b}}$ & $3,38 \pm 0,16^{\mathrm{a}}$ & 0,0001 \\
\hline Glicose $(\mu \mathrm{mol} / \mathrm{g})$ & $12,02 \pm 1,01$ & $10,45 \pm 1,21$ & $11,93 \pm 0,80$ & $12,64 \pm 1,21$ & NS \\
\hline Glicogênio ( $\mu$ mol glicose/g) & $28,44 \pm 6,18^{\mathrm{ab}}$ & $25,02 \pm 4,19^{b}$ & $27,16 \pm 2,89^{b}$ & $32,11 \pm 4,15^{\mathrm{a}}$ & 0,0355 \\
\hline TBARS (nmol MDA/mg proteína) & $17,75 \pm 6,42^{\mathrm{a}}$ & $11,55 \pm 5,46^{\mathrm{b}}$ & $9,44 \pm 2,59^{b}$ & $8,74 \pm 1,73^{b}$ & 0,0131 \\
\hline \multicolumn{6}{|c|}{10 dias de resfriamento } \\
\hline Amônia $(\mu \mathrm{mol} / \mathrm{g})$ & $1,44 \pm 0,10^{b}$ & $1,06 \pm 0,10^{c}$ & $1,42 \pm 0,07^{\mathrm{b}}$ & $2,32 \pm 0,09^{\mathrm{a}}$ & 0,0001 \\
\hline Glicose $(\mu \mathrm{mol} / \mathrm{g})$ & $4,82 \pm 0,88^{b}$ & $7,61 \pm 1,00^{\mathrm{a}}$ & $2,94 \pm 0,61^{\mathrm{b}}$ & $4,84 \pm 4,44^{\mathrm{b}}$ & 0,0022 \\
\hline Glicogênio ( $\mu \mathrm{mol}$ glicose/g) & $15,16 \pm 4,88$ & $12,28 \pm 5,82$ & $13,01 \pm 3,91$ & $14,13 \pm 4,26$ & NS \\
\hline TBARS (nmol MDA/mg proteína) & $3,37 \pm 0,80^{\mathrm{a}}$ & $1,71 \pm 0,51^{\mathrm{b}}$ & $1,87 \pm 0,32^{\mathrm{b}}$ & $2,07 \pm 1,06^{\mathrm{b}}$ & 0,0024 \\
\hline
\end{tabular}

Valores expressos como média \pm desvio padrão. Tratamentos com diferentes letras na mesma linha diferem significativamente de acordo com o teste de Duncan $(p<0,05)$. TBARS: substâncias reativas ao ácido tiobarbitúrico, MDA: malondialdeído, NS: não significativo. Fonte: Autores (2021).

Os lipídios em níveis elevados em produtos cárneos e similares são o principal determinante que provoca a degradação oxidativa (Jiang \& Xiong, 2016; Smaoui et al., 2016; Lahmar, Morcuende, Andrade, Chekir-Ghedira, \& Estévez, 2018), sendo denominada rancificação, gerando alteração de sabor, coloração, aroma e, portanto, dificultando a aceitabilidade e diminuindo o tempo hábil para o consumo (Smaoui et al. 2016; Pogorzelska et al., 2018).

A rancificação da gordura é causada por compostos químicos ou espécies reativas de oxigênio que causam quebra das ligações duplas nas frações fosfolipídicas das membranas celulares, mais frequente em peixes por possuírem maior grau de insaturação. Quando este processo ocorre é possível que haja formação de compostos químicos que levam à deterioração da carne hidroperóxidos, aldeídos e geração de produtos tóxicos secundários (Bekhit et al., 2021; Fogaça \& Sant’Ana, 2009; Prabhakar et al., 2020; Wu et al., 2019).

Estudos indicam a importância das vitaminas na dieta como antioxidante e a relação inversa da presença de compostos tóxicos no músculo. Tocher et al. (2002), observaram que menores quantidades de vitamina $\mathrm{E}$ nas dietas $(0,00 \mathrm{e} 0,01 \mathrm{~g} / \mathrm{kg}$, comparados à dieta com $0,11 \mathrm{~g} / \mathrm{kg}$ ) levavam à diminuição de seus níveis nos músculos e ao aumento da atividade oxidante do organismo, produzindo altos níveis de peróxidos lipídicos. Segundo Fogaça e Sant'Ana (2009), a prevenção ou diminuição da taxa de oxidação lipídica pode ser feita através da utilização de vitamina E nas dietas de organismos aquáticos, sendo comprovadamente eficiente na conservação de peixe durante o processamento e estocagem.

Matiucci e colaboradores (2021) utilizaram antioxidantes naturais durante a salga para a defumação de filés de tilápia e observaram efeito positivo na mitigação da oxidação durante o armazenamento do produto, corroborando com os resultados encontrados no presente estudo. Baseado nas determinações de TBARS, que chegaram a atingir um decréscimo em torno de $50 \%$, os resultados comprovam a importância do uso de antioxidantes, como cúrcuma e $\beta$-caroteno, que agiram como scavengers, removendo espécies reativas e diminuindo a ação das mesmas, contribuindo para melhor qualidade físico-química do filé. 
Para as demais variáveis bioquímicas, foi observada maior quantidade de amônia, aos cinco dias, nos tratamentos com antioxidantes quando comparados ao controle, com exceção da cúrcuma. Quando a quantidade de amônia está aumentada no músculo, isto sinaliza o início do processo de putrefação (Bekhit et al., 2021; Prabhakar et al., 2020; Wu et al., 2019). É possível que os diferentes antioxidantes atuem de diferentes formas, resultando em maior ou menor efetividade no controle de enzimas proteolíticas. O emprego do BHT resultou em uma quantidade de amônia superior quando comparado ao grupo controle e aos dois antioxidantes em investigação em ambos os períodos. Este achado respalda a utilização dos antioxidantes naturais avaliados no presente estudo nos processos industriais utilizados na cadeia produtiva de peixes.

No presente estudo o teor de glicogênio não apresentou diferença entre os tratamentos comparativamente ao grupo controle. Este polissacarídeo, presente na musculatura, é hidrolisado em glicose, que serve como substrato para a formação de ácido láctico, cujo acúmulo acarreta numa diminuição do pH e aumento da vida útil do peixe (Soares \& Gonçalves, 2012). Em relação à glicose, foi verificado que o filé que recebeu a adição de cúrcuma apresentou maiores níveis após 10 dias acondicionados.

A importância da inclusão de antioxidantes é retardar a deterioração do alimento e prorrogar o período no qual este está apropriado para consumo (Lahmar et al., 2018; Pogorzelska et al., 2018). A cúrcuma e o $\beta$-caroteno, utilizados no presente trabalho, foram eficientes assim como o BHT, que tradicionalmente é utilizado na indústria.

\section{Conclusão}

$\mathrm{O}$ aumento da demanda por produtos naturais faz com que o $\beta$-caroteno e a cúrcuma sejam opções adequadas como conservantes de filés de tilápia. Além de melhorar a vida útil do filé, agregar valor e incentivar o consumo, os mesmos proporcionam efeitos benéficos à saúde, contribuindo para a diminuição do efeito de radicais livres e espécies reativas que influenciam negativamente a saúde humana.

\section{Referências}

Alves, L. F. S., Corrêa, S. S., Rocha, J. D. M., Amado, D. A. V., Cottica, S. M., \& Souza, M. L. R. (2020). Use of natural antioxidants in sous vide tilapia fillet. Boletim de Indústria Animal, 77, 1-12.

Anvisa - Agência Nacional de Vigilância Sanitária. Regulamento Técnico: atribuição de função de aditivos e seus limites máximos de uso para a categoria 8 Carne e Produtos Cárneos 1998. Resolução GMC 73/97. Sobre Padrões de qualidade para alimentos. Resolução-RDC. Disponível em: http://portal.anvisa.gov.br/documents/33916/391619/Portaria+n\%C2\%BA+1004\%2C+de+11+de+dezembro+de+1998.pdf/41e1bc8f-b276-4022-9afbff0bb3c12c0c.

AOAC. Association of Official Analytical Chemists. Official methods of analysis. (18th ed.), Gaitherburg: Published by AOAC International. Washington DC, 2005.

Azambuja, C. R., Mattiazzi, J., Riffel, A. P. K., Finamor, I. A., Oliveira Garcia, L., Heldwein, C. G., Heinzmann, B. M., Baldisserotto, B., Pavanato, M. A., \& Llesuy, S. F. (2011). Effect of the essential oil of Lippia alba on oxidative stress parameters in silver catfish (Rhamdia quelen) subjected to transport. Aquaculture, 319(1-2), 156-161.

Battisti, E. K., Marasca, S., Durigon, E. G., Villes, V. S., Schneider, T. L., Uczay, J., Peixoto, N. C., \& Lazzari, R. (2017). Growth and oxidative parameters of Rhamdia quelen fed dietary levels of vitamin A. Aquaculture, 474, 11-17.

Bekhit, A. E-D. A., Holman, B. W. B., Giteru, S. G., \& Hopkins, D. L. (2021). Trends in Food Science \& Technology, 109, $280-302$.

Beraldo, J., Garcia, L. V., \& Marfori, T. G. (2020). Impacto da dieta mediterrânea e dieta low carb sobre a síndrome metabólica: uma revisão sistemática. Revista Ciência e Saúde On-line, 5(3), 19-30.

Bligh, E. G., \& Dyer W. J. (1959). A rapid method of total lipid extraction and purification. Canadian Journal of Biochemistry and Physiology, 37(8), 911917.

Buege, J. A., \& Aust, S. D. (1978). Microsomal lipid peroxidation. Methods in Enzymology, 52: 302-310.

Colpini, L. M. S., Castro, L. E. N., Durães, N. C., Carneiro, W. F., Tovo, A., Neto, \& Meurer, F. (2017). Valor nutricional do filé e carcaça de tilápias do Nilo alimentadas com rações contendo valores diversos de proteína e energia digestível. Revista Brasileira de Engenharia de Pesca, 10(2), $41-52$. 
Dias, J. A. R., Abreu, A. S., Silveira, D. S., Silva, A. S., Abe, H. A., Gomes, J. L. S., Barros, F. A. L., Silva, E. M., Cunha, F. S., Fujimoto, R. Y., \& Cordeiro, C. A. M. (2018). Uso de alecrim (Rosmarinus officinalis L.) no processo de defumação de peixes continentais. Revista Brasileira de Engenharia de Pesca, (1), $55-68$.

Dubois, M., Gilles, K. A., Hamilton, J. K., Rebers, P. A., \& Smith, F. (1956). Colorimetric method for the determination of sugars and related substances. Analytical Chemistry, 28(3), 350-356.

FAO. Food and Agriculture Organization of the United Nation. The state of world fisheries and aquaculture, 2020. Disponível em: < https://www.fao.org/3/ca9229en/ca9229en.pdf>.

Fogaça, F. H. S., \& Sant'Ana, L. S. (2009). Oxidação lipídica em peixes: mecanismo de ação e prevenção. Archives of Veterinary Science, $14(2)$, $117-127$.

Góes-Favon, S. P., Maricá, E. G. S., Shigematsu, E., \& Dorta, C. (2021). Microbial transglutaminase (MTGase) in the elaboration of fishburger using mechanically separated tilapia meat (Oreochromis niloticus) - Literature Review. Brazilian Journal of Technology, 4(1), 20-35.

Gonçalves, L. F., Mituuti, C. T., \& Haas, P. (2020). Efetividade da alimentação na prevenção do câncer de tireoide: revisão sistemática. Revista Brasileira de Cancerologia, 66(4): e-101072

Jiang, J., \& Xiong, Y. L. (2016). Natural antioxidants as food and feed additives to promote health benefits and quality of meat products: A review. Meat Science, 120, 107-117.

Lahmar, A., Morcuende, D., Andrade, M. J., Chekir-Ghedira, L., \& Estévez, M. (2018). Prolonging shelf life of lamb cutlets packed under high-oxygen modified atmosphere by spraying essential oils from North-African plants. Meat Science, 139, 56-64.

Machado, A. A., Peixoto, N. C., Hautrive, T. P., Uczay, J., \& Bezerra, A. S. (2020). Efeito antioxidante da Ilex paraguariensis e condimentos na oxidação lipídica de filés de Oreochromis niloticus. Revista Contexto \& Saúde, 20(39), 35-40.

Marchi, J. P., Tedesco, L., Cruz Melo, A., Frasson, A. C., França, V. F., Sato, S. W., \& Wietzikoski, E. C. (2016). Curcuma longa L., o açafrão da terra, e seus benefícios medicinais. Arquivos de Ciências da Saúde da UNIPAR, 20(3).

Matiucci, M. A., Rocha, J. D. M., Chambó, A. P. S., Alves, L. F. S., Silva, A. A., Oliveira, G. G., Corrêa, S. S., Silva, A. F., Gasparino, E., Goes, E. S. R., \& Souza, M. L. R. (2021). Ação antioxidante de pigmentos naturais em filés de tilápia do Nilo defumados. Research, Society and Development, 10(13), e79101320464-e79101320464.

Mesquita, G. F., \& Torquilho, H. S. (2016). O uso dos carotenóides para promoção da saúde. Perspectivas da Ciência e Tecnologia, 8(2), 1-28.

Moura, C. M. C., Costa, J. A., Sousa, A. M., Santos, J. H., Filho, Bacelar, R. G. A., Oliveira Santos, J. T., \& Muratori, M. C. S. (2018). Avaliação da qualidade microbiológica de filés de tilápia-do-Nilo (Oreochromis niloticus) e do gelo e a interação dos fatores após armazenagem. Medicina Veterinária (UFRPE), 12(1), 10-16

Nascimento, P. M. \& Scalabrini, H. M. (2020). Benefits of ômega 3 in cardiovascular disease prevention: integrative literature review. International Journal of Nutrology, 13, 95-101.

Neiva, C. R. P., Matsuda, C. S., Machado, T. M., Casarini, L. M., \& Tomita, R. Y. (2018). Glaciamento em filé de peixe congelado: revisão dos métodos para determinação de peso do produto. Boletim do Instituto de Pesca, 41(4), 899-906.

Oliveira, M. O. S, Luiz, D. B., Santos, V. R. V., Oliveira, E. H. S., \& Martins, G. A. S. (2019). Quality and safety aspects of the tambaqui (Colossoma macropomum) and painted from amazon (Pseudoplatystoma reticulatum x Leiarius marmoratus). Revista Desafios 6, 10-16.

Oliveira, N. D. M. S., Oliveira, W. R. M., Nascimento, L. C., Silva, J. M. S. F. D., Vicente, E., Fiorini, J. E., \& Bressan, M. C. (2008). Avaliação físicoquímica de filés de tilápia (Oreochromis niloticus) submetidos à sanitização. Food Science and Technology, 28, 83-89.

Olopade, O. A., Taiwo, I. O., Lamidi, A. A., \& Awonaike, O. A. (2016). Proximate composition of Nile tilapia (Oreochromis niloticus)(Linnaeus, 1758) and tilapia hybrid (red tilapia) from Oyan Lake, Nigeria. Buletin UASVM Food Science and Technology, 73(1), 19-23.

Pogorzelska, E., Godziszewska, J., Brodowska, M., \& Wierzbicka, A. (2018). Antioxidant potential of Haematococcus pluvialis extract rich in astaxanthin on colour and oxidative stability of raw ground pork meat during refrigerated storage. Meat Science, 135, 54-61.

Prabhakar, P. K., Vatsa, S., Srivastav, P. P., \& Pathak, S. S. (2020). A comprehensive review on freshness of fish and assessment: Analytical methods and recent innovations. Food Research International, 133, 1-17.

Schreiber, F. H. R., Zucatto, L. C., \& Lazzari, R. (2021). Canais de comercialização do pescado de água doce: um estudo em municípios da mesorregião Noroeste do Rio Grande do Sul. Research, Society and Development, 10(5), e50010514427-e50010514427.

Silva, E. S., Soares, F. M., Barros, J. R., \& Constant, P. B. L. (2019). Conservação de alimentos pelo uso de aditivos: uma revisão. Boletim do Centro de Pesquisa de Processamento de Alimentos, 37(2), 18-29.

Silva, I. C. G., Duarte, M. T. R., Landim, L. A. S. R., \& Duarte, C. T. T. (2020). Deficiency of vitamins and minerals: the role of technology in health prevention: an integrative review. Research, Society and Development, 9(10), e4129108700. https://doi.org/10.33448/rsd-v9i10.8700

Smaoui, S., Hsouna, A. B., Lahmar, A., Ennouri, K., Mtibaa-Chakchouk, A., Sellem, I., Najah, S., Bouaziz, M., \& Mellouli, L. (2016). Bio-preservative effect of the essential oil of the endemic Mentha piperita used alone and in combination with BacTN635 in stored minced beef meat. Meat Science, 117, 196-204.

Soares, K. M. P., \& Gonçalves, A. A. (2012). Qualidade e segurança do pescado. Revista do Instituto Adolfo Lutz, 71(1), 1-10. 
Research, Society and Development, v. 11, n. 1, e1411124136, 2022

(CC BY 4.0) | ISSN 2525-3409 | DOI: http://dx.doi.org/10.33448/rsd-v11i1.24136

Soares, L. A., Takeuti, T. D., Valeri, P. A. O., Silva, A. A., Lara, B. H. J., Terra-Júnior, J. A., Freitas, O., \& Crema, E. (2016). Impactos nutricionais da ingestão alimentar dos ácidos graxos ômega 3 de óleo de palma: uma revisão. Revista Brasileira de Obesidade, Nutrição e Emagrecimento, 10(56), 105-114.

Souza, J. T., Silva, É. L. B., Silva, L. R. C., Oliveira, P. V. C., Luz, K. S. S., Firmino, S. S., Pereira, G. S., Rebouças, L. O. S., \& Lima, P. O. (2020). Physicalchemical and sensory comparison of frozen fillets of Oreochromis niloticus and Pangasius hypophthalmus. Research, Society and Development, 9(10), e3489108583. https://doi.org/10.33448/rsd-v9i10.8583

Tocher, D. R., Mourente, G., Van der Eecken, A., Evjemo, J. O., Diaz, E., Bell, J. G., Geurden, I., Lavens, P., \& Olsen, Y. (2002). Effects of dietary vitamin E on antioxidant defence mechanisms of juvenile turbot (Scophthalmus maximus L.), halibut (Hippoglossus hippoglossus L.) and sea bream (Sparus aurata L.). Aquaculture Nutrition, 8(3), 195-207.

Vasconcelos, M. A. S., \& Melo, A. B., Filho. (2016). Conservação de alimentos. EDUFRPE.

Verdouw, H., Van Echteld, C. J. A., \& Dekkers, E. M. J. (1978). Ammonia determination based on indophenol formation with sodium salicylate. Water Research, 12(6), 399-402.

Viana, M. V., \& Arenari, V. S. (2019). Antioxidantes sintéticos utilizados em indústrias alimentícias e a possível substituição por antioxidantes naturais. Revista de Trabalhos Acadêmicos - Universo Campos dos Goytacazes, 12, 1-9.

Wu, L., Pu, H., \& Sun, D-W. (2019). Novel techniques for evaluating freshness quality attributes of fish: A review of recent developments. Trends in Food Science \& Technology, 83, 259-273. 